\title{
Dipyridamole echocardiography: the bedside stress test for coronary artery disease
}

\author{
Maurice B. Buchalter, John P. Bourke, Alyson Heads' and Terence Hawkins ${ }^{2}$ \\ Departments of Cardiology, ${ }^{1}$ Echocardiography and ${ }^{2}$ Medical Physics, Freeman Hospital, Newcastle upon \\ Tyne, UK.
}

Summary: Identification of dipyridamole-induced regional wall motion abnormalities by echocardiography has recently been proposed as an alternative diagnostic stress test for coronary artery disease. This study evaluates this new technique by comparing the results obtained (overall, regionally and by abnormality type) with those of thallium-201 myocardial imaging after dipyridamole stress in 25 patients.

Acceptable echocardiograms were obtained in 20 patients $(\mathbf{8 0} \%)$. Concordance of echocardiographic abnormalities for both overall and regional thallium abnormalities was $85 \%$. Sensitivity, specificity and predictive value of dipyridamole echocardiography for overall and regional thallium defects were $92 \%$, $71 \%$ and $85 \%$, and $91 \%, 81 \%$ and $85 \%$ respectively. However, concordance between the two for abnormality type (i.e. ischaemia versus infarction) was only $66 \%$ and the sensitivity, specificity and predictive value of dipyridamole echocardiography for identifying ischaemia as opposed to infarction were only $43 \%, 82 \%$ and $63 \%$, respectively.

There was substantial agreement between thallium and echocardiographic imaging after dipyridamole infusion in the diagnosis of coronary artery disease. Echocardiography appears less well able to distinguish infarction from active ischaemia. Dipyridamole echocardiography provides a highly versatile, noninvasive bedside stress test for the detection and localization of coronary artery disease.

\section{Introduction}

The versatility and clinical value of thallium-201 myocardial scintigraphy after dipyridamole infusion is now established in the diagnosis and assessment of patients with ischaemic heart disease. $^{1,2}$ Dipyridamole results in an increase in coronary artery flow by coronary arteriolar vasodilatation. Thallium is distributed to the coronary vascular beds in relation to blood flow and taken up by viable myocardial cells. ${ }^{3}$ Because coronary flow increases are limited in vascular beds supplied by a stenosed coronary artery, they appear as defects on the stress thallium images. Dipyridamole administration can also result in changes in regional wall motion which are different in normally perfused areas and those supplied by stenosed vessels. ${ }^{4,5}$ Echocardiography provides a convenient, sensitive and non-invasive means of assessing regional left ventricular wall motion $^{6}$ which is widely available in centres without access to thallium scintigraphy. It has recently been suggested that the combination of dipyridamole

Correspondence: M.B. Buchalter, M.B., M.R.C.P.I., Department of Clinical Pharmacology, Addenbrooke's Hospital, Hills Rd, Cambridge CB2 2QQ, UK.

Accepted: 8 February 1990 and echocardiography may be useful in evaluating patients with ischaemic heart disease. ${ }^{7,8}$

Studies to date have compared the dipyridamole echocardiographic technique with the results of coronary angiography. However, this is not the ideal comparison as the former examines the functional significance and the latter the anatomical severity of coronary stenoses. The aim of this study was to evaluate the role of the dipyridamoleecho test in the diagnosis of coronary artery disease by comparing it to another technique which also examines the functional significance of coronary artery lesions, namely thallium-201 imaging. We are thus comparing two non-invasive techniques which assess the presence and importance of any coronary artery lesions based on the functional consequences of those lesions. This will elucidate whether dipyridamole echocardiography is a useful clinical tool in the assessment of coronary artery disease.

\section{Materials and methods}

\section{Patient recruitment}

Twenty-five patients undergoing clinically indicated dipyridamole thallium imaging were entered into the study. Thirteen were known to 
have coronary artery disease and were undergoing thallium imaging to assess the extent of their disease and the remaining twelve were being imaged to establish whether their chest pain was due to coronary artery disease. The decision to seek consent was made without knowledge of the patients' clinical details or echocardiography suitability.

\section{Test procedure}

Immediately before and 5 minutes after dipyridamole infusion, two dimensional echocardiography was performed by the same experienced operator in all patients. Standard parasternal long and short axis and apical four chamber views were sought. After baseline electrocardiogram (ECG) and haemodynamics had been obtained, $0.56 \mathrm{mg} / \mathrm{kg}$ of dipyridamole was infused over 4 minutes into a large peripheral vein diluted to a total volume of $40 \mathrm{ml}$ in normal saline. ECG and haemodynamics were assessed every minute for 6 minutes. Intravenous thallous chloride (74 MBq) was injected 3 minutes after completion of the dipyridamole infusion. On completion of the second series of echocardiograms, thallium images $(128 \times 128$ matrix; $300 \mathrm{~K}$ counts) were obtained, using a gamma camera and computer system, in left anterior oblique, left lateral anterior projections. Redistribution images were obtained 3 hours later if any abnormality was seen on the initial study. Significant adverse effects of dipyridamole were reversed by intravenous aminophylline.

\section{Reporting}

\section{a. Thallium images}

Thallium images were reported by two experienced observers, reading together, blinded to the echocardiography results and initially blinded to the patients' clinical details. Myocardial regions were subdivided as shown in Figure 1. The coronary arterial supply of the respective segments has been schematically superimposed. After dipyridamole stress, myocardial segments were reported as having either normal or abnormal thallium uptake. Abnormal stress segments which normalized after redistribution ('reversing defects') were taken to indicate active ischaemia and those which stayed abnormal ('fixed defects') were interpreted as showing infarction.

\section{b. Echocardiograms}

These were reported by two experienced operators, reading together, blinded to both the clinical details and the thallium results. Myocardial regions were

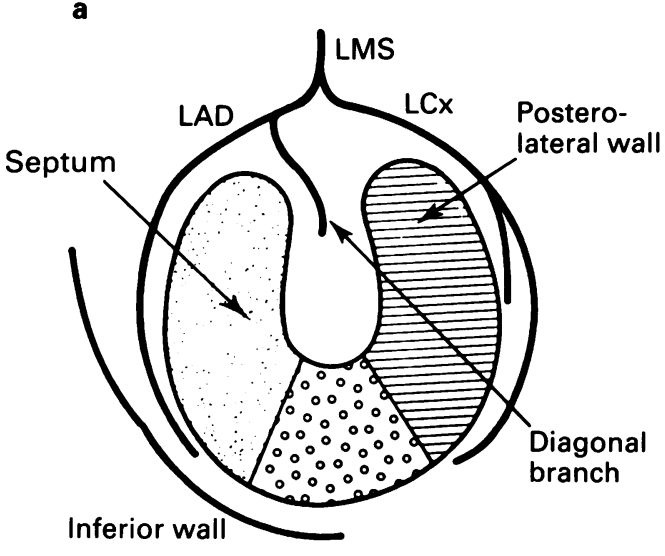

LEFT ANTERIOR OBLIQUE

b

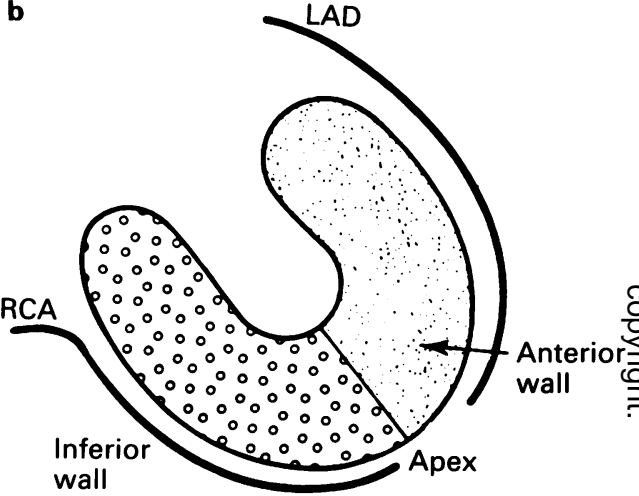

ANTERIOR

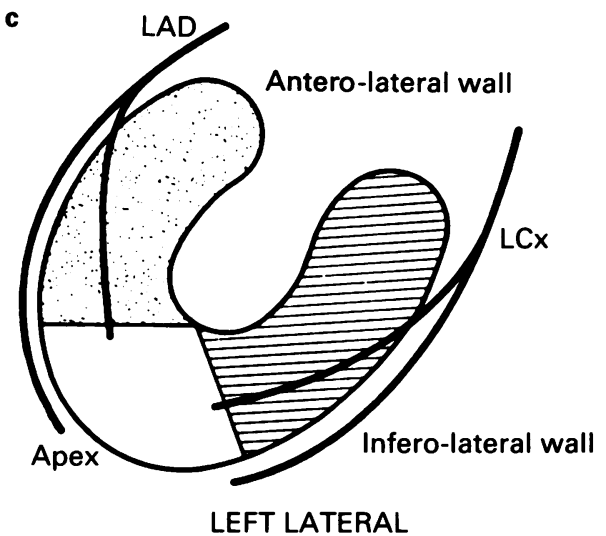

Figure 1 Schematic representation of the myocardial regions assessed on the thallium images which were compared to equivalent regions on the echo images. The arterial supply of each region is also indicated. The three standard views obtained for thallium imaging are shown.

subdivided as shown in Figure 2. Regional wall motion was scored both before and after the dipyridamole infusion as follows: 3 hyperdynamic, 

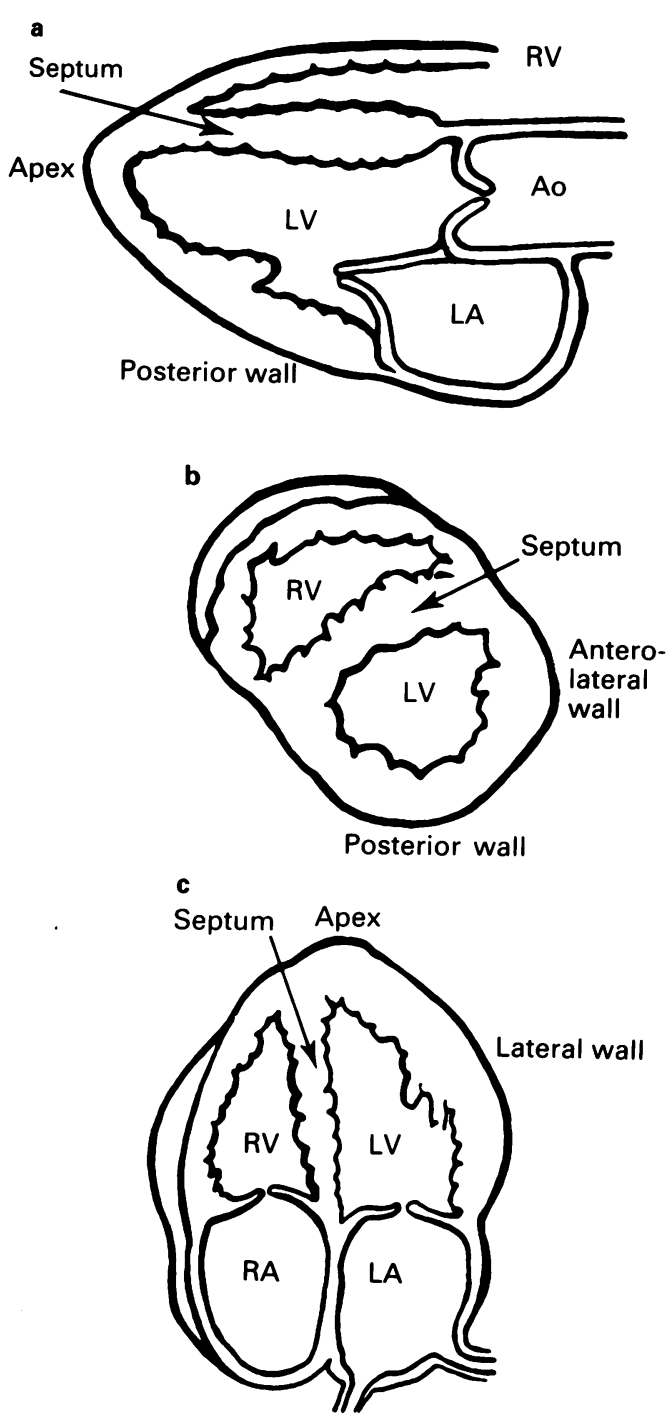

Figure 2 Schematic representation of myocardial regions assessed on the echo images which were compared to the equivalent regions on the thallium images. The three echo orientations obtained in all patients included in the analysis are shown (a, parasternal long axis; $b$, parasternal short axis; c, apical 4 chamber).

2 normal, 1 subnormal, 0 akinetic, -1 dyskinetic. The normal response to dipyridamole infusion is an increase in wall motion score from 2 to $3 .^{9} \mathrm{An}$ ischaemic response was defined as one with a normal score initially which either failed to increase or decreased after dipyridamole. Infarcted areas were those with an initially subnormal wall motion score.

\section{Dipyridamole-thallium versus dipyridamole-echo comparison}

Both sets of images were compared at three separate levels:

\section{(1) Overall comparison}

For each patient, both imaging techniques were designated as giving a positive (any abnormality) or negative (completely normal) result. The number of patients in whom both imaging techniques were either positive or negative (concordance) or one positive and one negative (discordance) were identified and the sensitivity, specificity and predictive value of the dipyridamole-echocardiography for dipyridamole-thallium defects calculated.

\section{(2) Region by region comparison}

Four regions of the left ventricle were considered separately - anterior, inferoposterior, lateral and apical. The first three correspond to the regions supplied by the left anterior descending, right coronary and circumflex arteries respectively. The apex has a variable arterial supply and was considered as a fourth region. A scan was considered to have a regional abnormality if any part of that region was abnormal. The number of segments for which the two scans were concordant (both giving the same result) or discordant (each giving a different result) was calculated and the sensitivity, specificity and predictive value of the echocardiographic technique for regional thallium defects calculated.

\section{(3) Abnormality type}

Regional abnormalities were assessed for either technique to determine whether ischaemia or infarction had been reported and the same parameters as for sections 1 and 2 above calculated.

\section{Results}

Twenty-five patients, 15 male, were enrolled into the study. Their mean age was 57.8 years (range 40-69). Seven patients had coronary angiography performed within 3 months of their non-invasive imaging.

After dipyridamole administration, mean heart rate rose ( 72 to 82 beats per minute; $P<0.01$ ) but mean blood pressure of the group did not change significantly (from $140 / 83 \mathrm{mmHg}$ to $140 / 81 \mathrm{mmHg}$; $P>0.1$ ). Two patients, however, became symptomatically hypotensive requiring dipyridamole reversal with aminophylline.

Technically satisfactory stress thallium images 
were obtained in all patients (75 images) and redistribution images, where indicated, were satisfactory in all but one patient ( 51 images). Complete echocardiographic assessments, both before and after dipyridamole, were obtained in 20 patients $(80 \%)$. A further two $(8 \%)$ had clinically useful echocardiography in limited views. In three patients $(12 \%)$ echocardiograms were too poor to allow meaningful interpretation. The 20 patients with comprehensive imaging by both techniques form the basis of the comparison.

Results of imaging comparisons are shown in Table I. While the sensitivity of the dipyridamoleechocardiographic technique for thallium defects is high for both overall $(93 \%)$ and regional $(91 \%)$ assessments, its specificity is lower at $71 \%$ and $81 \%$ respectively. Coronary angiograms were available in 2 of the 3 patients with discordant results in their global comparison. One patient had a left anterior descending artery stenosis, a normal thallium scan but an anterior lesion on the echo study. The second had normal coronary arteries, normal thallium but an abnormal echo. In the regional comparison, there were 9 areas identified by echocardiography as abnormal but with normal thallium uptake (i.e. 9 false positive regions).
Coronary angiography was available in 4 of these patients (5 segments) and showed that in 3 , the region was supplied by an artery with a significant stenosis, suggesting that the echocardiographic result was appropriate and in 2 there was no lesion suggesting the echo to be incorrect. Thus discordant results are explained by errors in each technique not just errors in the echo study.

\section{Discussion}

Thallium myocardial scintigraphy is well established in the diagnosis and assessment of patients with suspected ischaemic heart disease ${ }^{1,2}$ Thallium imaging after intravenous dipyridamole 'stress' provides a highly sensitive test in the evaluation of patients with coronary artery disease. However, its use is limited by the immobility and expense of the scanning equipment required.

The regional wall motion changes induced by dipyridamole are readily identified by echocardiography. ${ }^{4-6}$ The combination of dipyridamole and echocardiography, if clinically valuable, would provide a highly mobile, widely available, inexpensive and repeatable assessment of ischaemic heart

Table I Summary of results

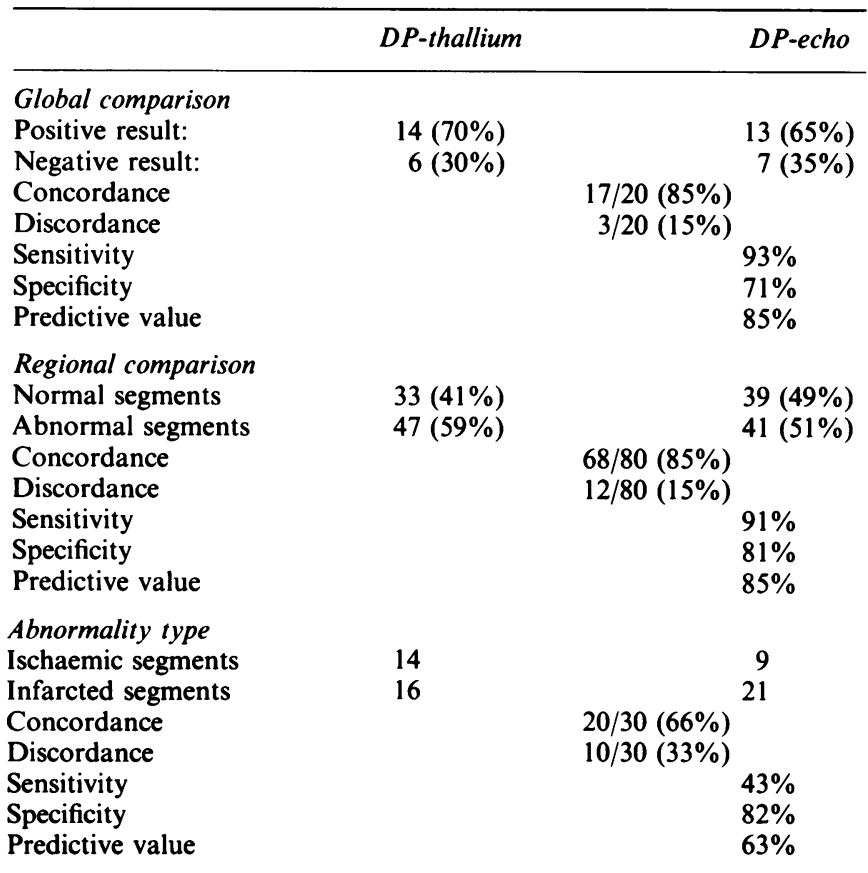

DP-thallium = dipyridamole thallium imaging; $D P$-echo $=$ dipyridamole echocardiography imaging.

Values indicate sensitivity, specificity and predictive value of DP-echo for DP-thallium. 
disease. This study was undertaken therefore to evaluate dipyridamole-echocardiography by comparing it with dipyridamole-thallium imaging, another technique which assesses the functional significance of coronary artery lesions.

The patients in this study were typical of those with coronary artery disease in not being ideal echocardiographic subjects. They are predominantly in an older age group and smokers with chronic lung disease. Despite this, interpretable echocardiographic images could be obtained in all views in $80 \%$. This suggests that most patients suspected of coronary artery disease would be suitable for this imaging technique.

The high concordance of the echocardiography with respect to thallium defects in both overall and regional analysis suggests that echocardiography is also an accurate predictor of coronary artery disease. We have found that for discordant results, it is sometimes the thallium and sometimes the echo that best compares with the presence of significant lesions at coronary angiography. The presence of significant atheroma does not necessarily imply a functional abnormality of course, and it is myocardial function that both these techniques assess. However, the angiographic data do suggest that the discordant results between the two techniques are not due to lesser accuracy of the echo but that they are each correct in different circumstances, possibly as they each assess different aspects of the functional consequences of coronary atheroma.

Previous studies of the dipyridamole-echocardiographic test have yielded differing results as regards its accuracy in diagnosing coronary artery disease. Picano et al. have reported sensitivity and specificity values of $60 \%$ and $100 \%$ respectively for echocardiography compared to angiography. ${ }^{7,8}$ Josephson et al., however, quote values of $89 \%$ and $71 \%$ - similar to those in this study. ${ }^{9}$ These differences are probably entirely due to the method of echocardiographic interpretation. Picano et al. define ischaemic segments as only those whose amplitude of movement reduces after dipyridamole stress, whereas Josephson and ourselves also include those segments which fail to increase their amplitude of movement. This wider definition increases the sensitivity of the technique with modest loss of specificity and is more suitable for the general assessment of patients with suspected coronary artery disease. In certain specific circumstances, however, such as assessing the functional significance of an arteriographic stenosis prior to angioplasty, the more stringent definition may be more appropriate.

When trying to distinguish ischaemic from infarcted myocardial segments, the sensitivity of the echocardiographic technique was poor. The definition used in this study required that initial wall motion be normal. However, some segments of viable muscle may be so compromised in their blood supply as to have a reduced amplitude of wall motion even at rest, so-called 'hibernating' myocardium. Such segments would be classified as infarcted by the terms of this study. This problem has not been addressed by studies to date. Further study is required to devise ways of identifying such segments without compromising the specificity of the diagnostic method.

This study demonstrates some clinical advantages of the dipyridamole-echocardiography as an alternative 'stress' test for coronary artery disease. It is both sensitive and specific, can be readily applied to the majority of patients and is less expensive than thallium imaging. The combination of the almost universal applicability of the dipyridamole 'stress' method and the convenience of echocardiography make dipyridamole-echocardiography a most valuable additional diagnostic aid in patients with suspected or proven coronary artery disease particularly in immobile patients and in centres without facilities for thallium scintigraphy.

\section{References}

1. Leppo, J.A., Boucher, C.A., Okade, R.D., Newell, J.B., Strauss, H.W. \& Pohost, G.H. Serial thallium-201 myocardial imaging after dipyridamole infusion: diagnostic utility in detecting coronary stenoses and relationship to regional wall motion. Circulation 1982, 66: 649-657.

2. Reisman, S. Dipyridamole thallium testing. An alternative form of stress testing in patients unable to exercise. Chest 1985 , 88: $321-322$.

3. Feldman, R.L., Nichols, W.W., Pepine, C.J. \& Conti, C.R. Acute effects of intravenous dipyridamole on regional coronary hemodynamics and metabolism. Circulation 1981, 64: 333-334.

4. Iskandrian, A.S., Heo, J., Askenase, A., Segal, B. \& Auerbach, N. Dipyridamole cardiac imaging. Am Heart $J$ 1988, 115: 432-442.

5. Distante, A., Roval, D., Picano, E. et al. Transient changes in left ventricular mechanics during attacks of Prinzmetal angina: a B-mode echocardiographic study. Am Heart J 1984, 108: 441-446.

6. Mason, S.K., Weiss, J.L., Weisfeldt, M., Garrison, J.B. \& Fortuin, N.J. Exercise echocardiography; detection of wall motion abnormalities during ischemia. Circulation 1979, 59: $50-59$.

7. Picano, E., Masini, M., Distante, A. et al. Dipyridamoleechocardiography test in patients with exercise induced ST. segment elevation. Am J Cardiol 1986, 57: 765-768.

8. Picano, E., Distante, A., Masini, M., Morales, M.A., Lattanzi, F. \& L'Abbate, A. Dipyridamole-echocardiography test in effort angina pectoris. Am J Cardiol 1985, 56: 452-456.

9. Josephson, R.A., Weiss, J.L., Flaherty, J.T., Ouyang, P. \& Shapiro, E.P. Dipyridamole-echocardiography detects vulnerable myocardium in the early post-infarct period. Circulation 1986, 74 (Suppl) II: 469. 